\title{
Beam section stiffness properties using a single layer of 3D solid elements
}

\author{
Couturier, Philippe; Krenk, Steen; Høgsberg, Jan Becker
}

Published in:

Computers and Structures

Link to article, DOI:

10.1016/j.compstruc.2015.03.010

Publication date:

2015

Document Version

Peer reviewed version

Link back to DTU Orbit

Citation (APA):

Couturier, P., Krenk, S., \& Høgsberg, J. B. (2015). Beam section stiffness properties using a single layer of 3D solid elements. Computers and Structures, 156, 122-133. https://doi.org/10.1016/j.compstruc.2015.03.010

\section{General rights}

Copyright and moral rights for the publications made accessible in the public portal are retained by the authors and/or other copyright owners and it is a condition of accessing publications that users recognise and abide by the legal requirements associated with these rights.

- Users may download and print one copy of any publication from the public portal for the purpose of private study or research.

- You may not further distribute the material or use it for any profit-making activity or commercial gain

- You may freely distribute the URL identifying the publication in the public portal 


\title{
Beam Section Stiffness Properties using a Single Layer of 3D Solid Elements
}

\author{
Philippe J. Couturier ${ }^{1,2}$, Steen Krenk ${ }^{1}$, and Jan Høgsberg ${ }^{1}$ \\ ${ }^{1)}$ Department of Mechanical Engineering, \\ Technical University of Denmark, DK-2800 Lyngby, Denmark \\ ${ }^{2)}$ Siemens Wind Power, DK-7330 Brande, Denmark
}

\begin{abstract}
A method is presented for analysis of the properties of general cross-sections with arbitrary geometry and material distribution. The full six by six cross-section stiffness matrix is evaluated from a single element thickness slice represented by 3D solid elements with lengthwise Hermitian interpolation with six independent imposed deformation modes corresponding to extension, torsion, bending and shear. The flexibility matrix of the slice is obtained from complementary elastic energy, and the stiffness matrix is obtained by extracting and inverting the cross-section flexibility. Three examples illustrate the accuracy of the method for solid and thin-walled sections with isotropic and general anisotropic materials.
\end{abstract}

Keywords: Cross-section analysis, beam stiffness parameter, finite element method.

\section{Introduction}

Rotor blades of wind turbines have increased considerably in size in recent years making them more flexible in relation to the operational loads. To meet deflection, fatigue and performance requirements, they must be designed with complex cross-section geometries made up of composite fiber materials. For most of the design process, full three-dimensional Finite Element analysis is computationally expensive and does not lend itself well to design space exploration and the analysis of numerous load scenarios. However, because of the slender nature of rotor 
blades their behaviour can accurately and effectively be predicted using beam models. In a beam approximation, the three-dimensional elasticity problem is reduced to a one-dimensional structural problem. Irrespective of the beam theory used to model the blade, the cross-section properties constitute an essential part of the beam model.

Rotor blade cross-sectional analysis methodology can generally be grouped in two categories based on whether a centerline approach or a Finite Element discretization is used $[1,2,3]$. Methods based on a centerline approach have been shown to be very efficient and provide satisfactory results for many thin-walled cross-sections. However, the centerline approach is sensitive to relative wall curvature, shear-flow representation at corners in the cross-section layout, and limitations regarding transverse displacement effects. Because of these limitations, approaches which model beams with complex geometries and general anisotropic materials rely on Finite Element discretization. The most widely recognized of these approaches in the field of rotor blade modelling is the Variational Asymptotic Beam Sectional Analysis (VABS) based on the theory developed by Hodges and $\mathrm{Yu}[4,5]$. In this procedure the beam is considered as a three dimensional body described by a lengthwise variation of properties associated with crosssections. It results in a split of the elasticity problem into a two-dimensional linear cross-sectional analysis and a one-dimensional beam analysis. In the cross-section problem the displacements are represented by isoparametric $2 \mathrm{D}$ elements.

Another finite element based approach, which has been shown to accurately extract general cross-sectional properties of many beam structures, was developed by Giavotto et al. [6, 7]. The theory is based on defining two types of solutions of a virtual work formulation of which the non-decaying solution is used to recover the cross-section stiffness properties. The theory was implemented in two computer codes, namely Anisotropic Beam Analysis (ANBA) developed by the original authors and more recently in Beam Cross section Analysis Software (BECAS) developed by Blasques [8]. Both the VABS and the ANBA theories reduce the inherent three dimensional nature of the problem to a two dimensional form.

This paper builds on the concept of six equilibrium states of a beam previously used by 
Krenk and Jeppesen [9], Høgsberg and Krenk [10] and Jonnalagadda and Whitcomb [11] for the analysis of cross-sections made of orthotropic materials. The theories developed in these papers are restricted to orthotropic material properties and include restrictive assumptions regarding the detailed distribution of deformations and stresses over the cross-section. In the present method, the cross-section stiffness matrix is calculated based on the analysis of a slice of the beam in the form of a single layer of elements with cubic shape-function variation in the length-wise direction and with arbitrary anisotropic properties. Six independent equilibrium deformation modes corresponding to extension, torsion, homogeneous bending and homogeneous shear are generated by imposing suitable displacement increments across the slice via Lagrange multipliers. Elastic energy equivalence of the 3D slice and the complementary elastic energy calculated in terms of the internal force/moment distribution is then used to define the full six by six flexibility matrix of the cross-section. Finally, the corresponding cross-section stiffness matrix follows by inversion of this flexibility matrix.

The advantages of the present finite-thickness slice method are that it avoids the development of any special 2D theory for the stress and strain distributions over the cross-section and enables a simple and direct representation of material discontinuities and general anisotropy via their well-established representation in 3D elements. Elements with cubic Hermitian lengthwise interpolation in combination with linear, quadratic and cubic in-plane interpolation have been implemented. Combination of these interpolation types enables accurate modelling of thin-walled parts, lamina built-up parts, as well as more massive parts.

\section{Beam statics description}

Consider a beam of length $l$ with longitudinal coordinate $x_{3}$ and cross-section coordinates $x_{1}$ and $x_{2}$ as shown in Fig. 1(a). The origin is located at the center cross-section plane of the beam, whereby the front and back of the beam are located at $x_{3}=l / 2$ and $x_{3}=-l / 2$, respectively. The beam supports the equilibrium states of tension, torsion, bending, and shear. 


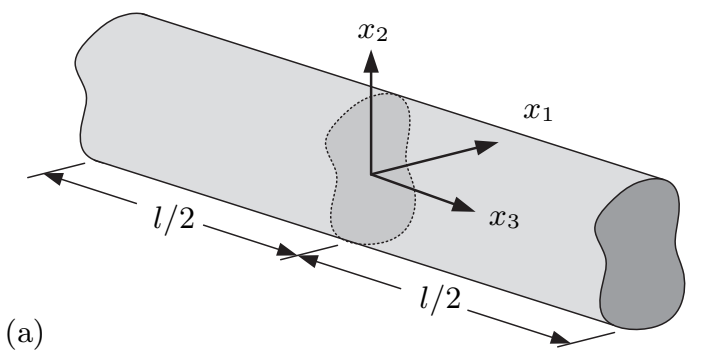

(b)

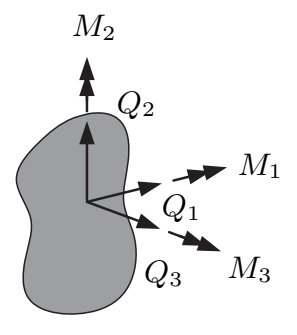

Figure 1: (a) Coordinate system, (b) Section forces and moments.

The static states of a beam are described by three forces and three moments at each crosssection along $x_{3}$ which are statically equivalent to the exact distribution of the in-plane stresses. These six forces and moments are grouped together in the generalized force vector $\mathbf{q}\left(x_{3}\right)=$ $\left[Q_{1}\left(x_{3}\right) Q_{2}\left(x_{3}\right) Q_{3}\left(x_{3}\right) M_{1}\left(x_{3}\right) M_{2}\left(x_{3}\right) M_{3}\left(x_{3}\right)\right]^{T}$. The components $Q_{1}\left(x_{3}\right)$ and $Q_{2}\left(x_{3}\right)$ are two shear forces, and $Q_{3}\left(x_{3}\right)$ is the axial force. The components $M_{1}\left(x_{3}\right)$ and $M_{2}\left(x_{3}\right)$ are bending moments, and $M_{3}\left(x_{3}\right)$ is the torsion moment component with respect to the origin of the reference coordinate system. A compact notation is achieved when representing the two in-plane directions using Greek subscripts $\alpha, \beta=1,2$ which allows summation over repeated Greek subscripts. This notation includes use of the two-dimensional permutation symbol $e_{\alpha \beta}$ which takes the following values based on the indices $e_{12}=1, e_{21}=-1$, and $e_{11}=e_{22}=0$. Using the Greek subscripts and the permutation symbol, internal forces and moments are defined in terms of stresses on a cross-section as

$$
\begin{aligned}
Q_{\alpha}\left(x_{3}\right) & =\int_{A} \sigma_{3 \alpha} d A, & M_{\alpha}\left(x_{3}\right) & =\int_{A} e_{\alpha \beta} x_{\beta} \sigma_{3} d A, \\
Q_{3}\left(x_{3}\right) & =\int_{A} \sigma_{3} d A, & M_{3}\left(x_{3}\right) & =\int_{A} x_{\alpha} e_{\alpha \beta} \sigma_{3 \beta} d A .
\end{aligned}
$$

The internal force and moment components are illustrated in Fig. 1(b).

\subsection{Equilibrium states}

The statics of a beam is described by six equilibrium states, namely the homogeneous states of tension, torsion, bending, and shear. The case of homogeneous tension is illustrated in Fig. 2(a). 


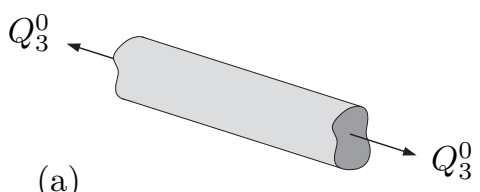

(a)

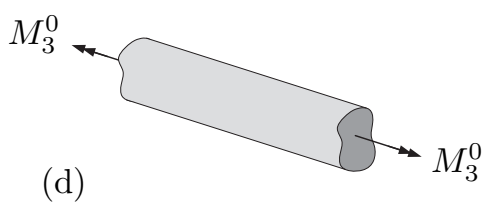

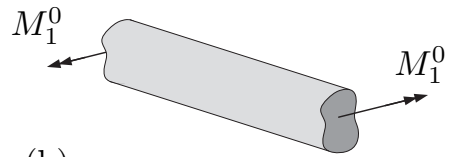

(b)

(e)

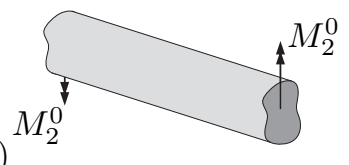

(c)

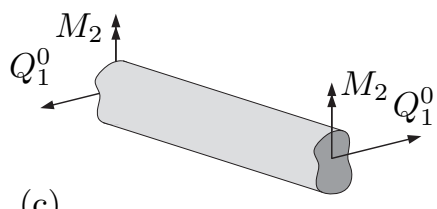

(f)

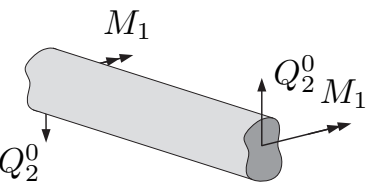

Figure 2: Six equilibrium states: (a) Tension, (d) Torsion, (b,e) Bending, (c,f) Shear.

Opposing axial forces of magnitude $Q_{3}^{0}$ are acting at the ends of the beam. Similarly the case of homogeneous torsion illustrated in Fig. 2(d) is characterized by opposing torsion moments of magnitude $M_{3}^{0}$ acting at the ends of the beam. The homogeneous bending states are illustrated in Fig. 2(b) and (e). Opposing bending moments of magnitude $M_{\alpha}^{0}$ act at the ends of the beam. Finally, the states of homogeneous shear are illustrated in Fig. 2(e) and (f). Here, opposing shear forces of magnitude $Q_{\alpha}^{0}$ are applied to the ends of the beam. This results in a total external moment that is counteracted by identical bending moments at the end-sections equal to

$$
M_{\alpha}\left( \pm \frac{1}{2} l\right)=\frac{1}{2} l e_{\alpha \beta} Q_{\beta}^{0}
$$

From equilibrium considerations a beam without external loads will exhibit constant internal normal force, shear forces and torsion moment, while the bending moments will vary linearly with the shear force as gradient. The six equilibrium states are therefore fully defined by the

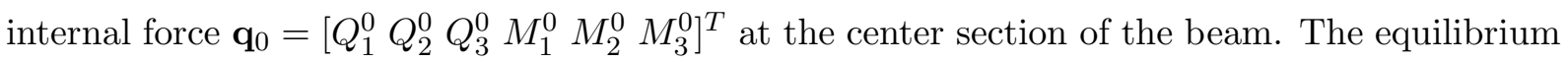


distribution of internal forces in the beam in terms of $\mathbf{q}_{0}$ follows as

$$
\left[\begin{array}{l}
Q_{1}\left(x_{3}\right) \\
Q_{2}\left(x_{3}\right) \\
Q_{3}\left(x_{3}\right) \\
M_{1}\left(x_{3}\right) \\
M_{2}\left(x_{3}\right) \\
M_{3}\left(x_{3}\right)
\end{array}\right]=\left[\begin{array}{cccccc}
1 & 0 & 0 & 0 & 0 & 0 \\
0 & 1 & 0 & 0 & 0 & 0 \\
0 & 0 & 1 & 0 & 0 & 0 \\
0 & x_{3} & 0 & 1 & 0 & 0 \\
-x_{3} & 0 & 0 & 0 & 1 & 0 \\
0 & 0 & 0 & 0 & 0 & 1
\end{array}\right]\left[\begin{array}{c}
Q_{1}^{0} \\
Q_{2}^{0} \\
Q_{3}^{0} \\
M_{1}^{0} \\
M_{2}^{0} \\
M_{3}^{0}
\end{array}\right]
$$

The distribution of internal forces written in a more compact notation becomes

$$
\mathbf{q}\left(x_{3}\right)=\mathbf{T}\left(x_{3}\right) \mathbf{q}_{0},
$$

where the interpolation matrix $\mathbf{T}\left(x_{3}\right)$ is defined in (3).

\subsection{Flexibility matrix}

Following the general formulation of equilibrium based beam elements in [12], the deformation associated with the internal forces and moments $\mathbf{q}\left(x_{3}\right)$ is described in terms of six strains defined by the strain vector $\gamma\left(x_{3}\right)=\left[\begin{array}{llllll}\varepsilon_{1} & \varepsilon_{2} & \varepsilon_{3} & \kappa_{1} & \kappa_{2} & \kappa_{3}\end{array}\right]^{T}$. The components $\varepsilon_{1}$ and $\varepsilon_{2}$ are generalized shear strains, and $\varepsilon_{3}$ is the axial strain. Similarly $\kappa_{1}$ and $\kappa_{2}$ are the components of bending curvature, while $\kappa_{3}$ is the rate of twist. The generalized strain vector $\gamma\left(x_{3}\right)$ is defined such that it is conjugate to the internal force vector $\mathbf{q}\left(x_{3}\right)$ with respect to energy. Thus, the specific elastic energy associated with a cross-section is given as

$$
W_{s}\left(x_{3}\right)=\frac{1}{2} \gamma\left(x_{3}\right)^{T} \mathbf{q}\left(x_{3}\right) .
$$

For linear elastic beams there is a linear relation between the internal forces and the conjugate strains. This relation can be written either in flexibility or stiffness format,

$$
\gamma\left(x_{3}\right)=\mathbf{C} \mathbf{q}\left(x_{3}\right), \quad \mathbf{q}\left(x_{3}\right)=\mathbf{D} \gamma\left(x_{3}\right)
$$


where $\mathbf{C}$ and $\mathbf{D}=\mathbf{C}^{-1}$ are the cross-section flexibility and stiffness matrix, respectively. Both are six by six symmetric matrices and as such can contain up to twenty-one independent entries in the case of a fully general anisotropic cross-section without geometric or material symmetries. In this case, for example, the principal axes of shear and bending may be different, and torsion may couple to extension and bending. Considering a beam with constant cross-section properties $\mathbf{C}$ in the longitudinal direction and eliminating $\gamma$ in (5) using (6), the following representation of the energy per unit length at $x_{3}$ expressed in terms of the cross-section flexibility matrix is obtained

$$
W_{s}\left(x_{3}\right)=\frac{1}{2} \mathbf{q}\left(x_{3}\right)^{T} \mathbf{C} \mathbf{q}\left(x_{3}\right)
$$

The flexibility matrix of the equilibrium states of the beam follows from integration of the cross-section flexibility relation (7) over the beam length

$$
W_{e}=\int_{-l / 2}^{l / 2} W_{s}\left(x_{3}\right) d x_{3}=\int_{-l / 2}^{l / 2} \frac{1}{2} \mathbf{q}\left(x_{3}\right)^{T} \mathbf{C} \mathbf{q}\left(x_{3}\right) d x_{3} .
$$

The energy $W_{e}$ can be represented in terms of the internal forces and moments by the centersection values $\mathbf{q}_{0}$ by (3) as

$$
W_{e}=\frac{1}{2} \mathbf{q}_{0}^{T} \mathbf{H} \mathbf{q}_{0}
$$

where the beam flexibility matrix $\mathbf{H}$ corresponding to the six equilibrium states is defined by the integral

$$
\mathbf{H}=\int_{-l / 2}^{l / 2} \mathbf{T}\left(x_{3}\right)^{T} \mathbf{C} \mathbf{T}\left(x_{3}\right) d x_{3} .
$$

Carrying out the $x_{3}$-integration in explicit form with $\mathbf{T}\left(x_{3}\right)$ given by the matrix in (3) provides 
the relation between the beam flexibility matrix $\mathbf{H}$ and the cross-section flexibility matrix $\mathbf{C}$,

$$
\mathbf{H}=l\left[\begin{array}{cccccc}
C_{11}+\frac{1}{12} l^{2} C_{55} & C_{12}-\frac{1}{12} l^{2} C_{54} & C_{13} & C_{14} & C_{15} & C_{16} \\
C_{21}-\frac{1}{12} l^{2} C_{45} & C_{22}+\frac{1}{12} l^{2} C_{44} & C_{23} & C_{24} & C_{25} & C_{26} \\
C_{31} & C_{32} & C_{33} & C_{34} & C_{35} & C_{36} \\
C_{41} & C_{42} & C_{43} & C_{44} & C_{45} & C_{46} \\
C_{51} & C_{52} & C_{53} & C_{54} & C_{55} & C_{56} \\
C_{61} & C_{62} & C_{63} & C_{64} & C_{65} & C_{66}
\end{array}\right] .
$$

It is seen how the bending flexibility parameters $C_{55}, C_{44}, C_{45}$, and $C_{54}$ of the cross-section enter the constant shear modes of the beam due to their linearly varying bending moment.

\subsection{Energy equivalence}

The beam displacement field representation with respect to $x_{3}$ varies at most as a third degree polynomial. In what follows the solution to this displacement field is defined using a Hermitian representation in terms of the displacement and displacement derivative fields on the front $(+)$ and back (-) faces of the beam as shown in Fig. 3. The implementation of the Hermitian interpolation is presented in Appendix A. The displacement vector field $\mathbf{u}(\mathbf{x})=\left[u_{1}, u_{2}, u_{3}\right]^{T}$ is described in terms of the the coordinates $\mathbf{x}=\left[x_{1}, x_{2}, x_{3}\right]^{T}$ by the representation

$$
\mathbf{u}(\mathbf{x})=\mathbf{N}(\mathbf{x}) \mathbf{v}
$$

The matrix $\mathbf{N}(\mathbf{x})$ contains the shape functions corresponding to the generalized nodal displacements contained in the column vector $\mathbf{v}=\left[\tilde{\mathbf{v}}_{1}, \ldots, \tilde{\mathbf{v}}_{2 m}\right]^{T}$ where $m$ is the number of nodes on each side of the slice. The six nodal degrees of freedom are defined as $\tilde{\mathbf{v}}_{i}=\left[u_{\alpha}, u_{3}, u_{\alpha}^{\prime}, u_{3}^{\prime}\right]$, where the components $u_{\alpha}$ and $u_{3}$ represent the in-plane and axial displacements, respectively, while the components $u_{\alpha}^{\prime}$ and $u_{3}^{\prime}$ represent the corresponding derivatives with respect to the axial coordinate $x_{3}$. The displacement vector $\mathbf{v}$ is conjugate to the force vector $\mathbf{p}=\left[\tilde{\mathbf{p}}_{1}, \ldots, \tilde{\mathbf{p}}_{2 m}\right]^{T}$, with the nodal forces defined as $\tilde{\mathbf{p}}_{i}=\left[f_{\alpha}, f_{3}, f_{\alpha}^{\prime}, f_{3}^{\prime}\right]$. The components $f_{\alpha}$ and $f_{3}$ represent the 


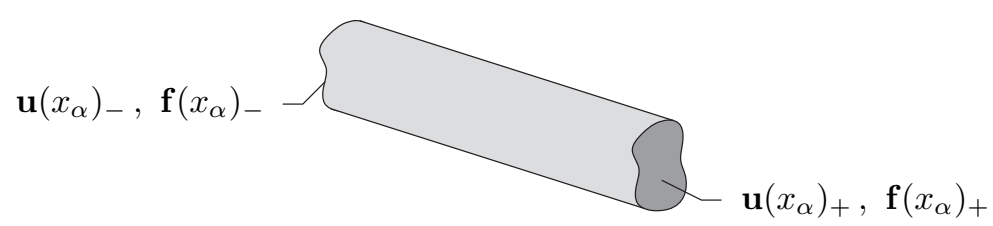

Figure 3: Degrees of freedom and static components at the front $(+)$ and the back $(-)$.

three force components, while the components $f_{\alpha}^{\prime}$ and $f_{3}^{\prime}$ represent three moment components conjugate to the displacement derivatives $u_{\alpha}^{\prime}$ and $u_{3}^{\prime}$, respectively.

The procedure consists in solving the finite element problem corresponding to six independent equilibrium states, e.g. those illustrated in Fig. 2. The generalized nodal displacements $\mathbf{v}_{j}$ and conjugate generalized nodal forces $\mathbf{p}_{j}$ for $j=1, \cdots, 6$ are arranged as columns in the following two matrices

$$
\mathbf{V}=\left[\mathbf{v}_{1}, \ldots, \mathbf{v}_{6}\right], \quad \mathbf{P}=\left[\mathbf{p}_{1}, \ldots, \mathbf{p}_{6}\right]
$$

The solution $\mathbf{v}_{j}, \mathbf{p}_{j}$ for each of the states are calculated by imposing appropriate displacements on the end-sections of the beam slice, as discussed in Section 3. Once a solution has been calculated, the section-force component vector $\mathbf{q}_{0 j}$ at the center section is evaluated. These six center section-force vectors are arranged in the matrix

$$
\mathbf{R}=\left[\mathbf{q}_{01}, \ldots, \mathbf{q}_{06}\right]
$$

When the six equilibrium states are linearly independent the matrix $\mathbf{R}$ is regular and can be inverted.

A general equilibrium state can now be represented as a linear combination of the six equilibrium states introduced above. The components of this representation are denoted $\mathbf{s}=$ $\left[s_{1}, \cdots, s_{6}\right]^{T}$, whereby the nodal displacements and nodal forces take the form

$$
\mathbf{v}=\sum_{j=1}^{6} \mathbf{v}_{j} s_{j}=\mathbf{V} \mathbf{s}, \quad \mathbf{p}=\sum_{j=1}^{6} \mathbf{p}_{j} s_{j}=\mathbf{P} \mathbf{s} .
$$


The section-forces of the center section are expressed similarly as

$$
\mathbf{q}_{0}=\sum_{j=1}^{6} \mathbf{q}_{0 j} s_{j}=\mathbf{R} \mathbf{s} .
$$

The elastic energy of the slice can now be expressed alternatively in terms of the flexibility matrix $\mathbf{H}$ by use of (9) or as the product of the nodal forces and displacements as given by the representations in (15),

$$
W_{e}=\frac{1}{2} \mathbf{s}^{T} \mathbf{R}^{T} \mathbf{H} \mathbf{R} \mathbf{s}=\frac{1}{2} \mathbf{s}^{T} \mathbf{V}^{T} \mathbf{P} \mathbf{s}
$$

The matrix $\mathbf{R}$ is regular due to the assumed independence of the imposed displacement conditions, and thus the energy can be expressed in terms of the internal force components $\mathbf{q}_{0}$ at the center cross-section. Elimination of the parameters $\mathbf{s}$ by use of (16) gives

$$
W_{e}=\frac{1}{2} \mathbf{q}_{0}^{T} \mathbf{H} \mathbf{q}_{0}=\frac{1}{2} \mathbf{q}_{0}^{T} \mathbf{R}^{-T}\left(\mathbf{V}^{T} \mathbf{P}\right) \mathbf{R}^{-1} \mathbf{q}_{0}
$$

It follows from this result that the columns of the matrix $\mathbf{V R}^{-1}$ contains the nodal displacement vectors of the six equilibrium states corresponding to a unit value of the corresponding internal force $\mathbf{q}_{0}$ at the center cross-section, while the nodal forces of these six equilibrium states are contained in the columns of the matrix $\mathbf{P} \mathbf{R}^{-1}$.

By Betti's result of equality of the work of one equilibrium state of stresses through a different set of equilibrium displacements with the work obtained when interchanging the roles of the two states it follows that the matrix $\mathbf{V}^{T} \mathbf{P}$ is symmetric, and thus the flexibility matrix $\mathbf{H}$ is obtained in the form

$$
\mathbf{H}=\mathbf{R}^{-T}\left(\mathbf{V}^{T} \mathbf{P}\right) \mathbf{R}^{-1}
$$

In particular, for orthotropic beams with symmetric cross-section the basic deformation cases are conveniently chosen in accordance with this symmetry, leading to a diagonal matrix $\mathbf{R}$ corresponding to a simple normalization of the imposed deformation states.

The stiffness matrix of a beam element, formulated in terms of the displacements and rotations at the beam ends, is easily obtained explicitly in terms of the inverse $\mathbf{H}^{-1}$ as described in $[12]$. 
(a)

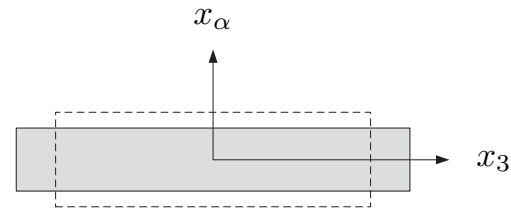

(c)

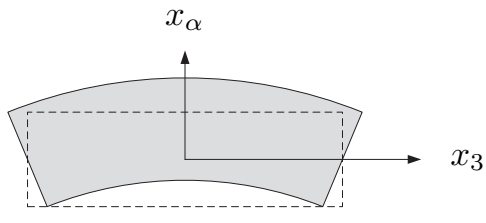

(b)

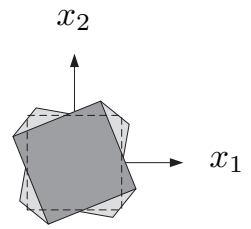

(d)

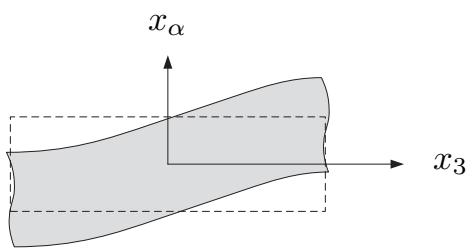

Figure 4: Deformation modes: (a) Extension, (b) Twist, (c) Bending, (d) Shear.

\section{Imposed deformation modes}

The six independent equilibrium states are chosen as the deformation modes corresponding to extension, twist, bending, and shear. These deformation modes are illustrated in Fig. 4 for the case of a square orthotropic cross-section where the geometry before deformation is sketched using dotted lines. The equilibrium states are defined in terms of differences in forces and moments at the front and back face of the beam. It is therefore convenient to group the nodal displacements at the front $(+)$ and back $(-)$ faces as

$$
\mathbf{v}_{ \pm}=\mathbf{v}\left( \pm \frac{1}{2} l\right)
$$

from which the nodal displacements can be split into an increment and a mean value across the length of the beam,

$$
\Delta \mathbf{v}=\mathbf{v}_{+}-\mathbf{v}_{-}, \quad 2 \overline{\mathbf{v}}=\mathbf{v}_{+}+\mathbf{v}_{-} .
$$

The six displacement modes are generated by imposing suitable displacement increments defined in terms of $\Delta \mathbf{v}$, while rigid body motion is constrained by using the mean displacements $\overline{\mathbf{v}}$.

It is convenient to describe the nodal displacement increment $\Delta \mathbf{v}$ of the six deformation

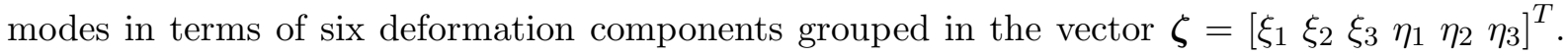


The components $\xi_{1}$ and $\xi_{2}$ are associated with the displacement increments of the two shear modes, and $\xi_{3}$ with the displacement increments of the extension mode. Similarly $\eta_{1}$ and $\eta_{2}$ are associated with the displacement increments of the two bending modes, and $\eta_{3}$ with the displacement increments of the twist mode. Each of the deformation modes corresponds to activating one of the components of $\boldsymbol{\zeta}$ while setting the remaining five to zero.

\subsection{Extension mode}

The extension deformation mode illustrated in Fig. 4(a) is described by an elongation of the beam equal to $\xi_{3}$. No internal generalized shear forces $Q_{\alpha}$ occur in this mode, which yields constant forces and moments along the longitudinal axis, $\mathbf{q}\left(x_{3}\right)=\mathbf{q}_{0}$. This leads to a uniform transverse contraction along the beam and thereby the in-plane displacement increment $\Delta u_{\alpha}\left(x_{\gamma}\right)$ for each node pair is zero. The displacement increments defining the extension deformation mode therefore follow as

$$
\Delta u_{\alpha}\left(x_{\gamma}\right)=0, \quad \Delta u_{3}\left(x_{\gamma}\right)=\xi_{3}, \quad \text { Node pairs } i=1, \ldots, m .
$$

\subsection{Twist mode}

The twist deformation mode illustrated in Fig. 4(b) is defined by a constant rate of twist $\eta_{3}$ about the $x_{3}$-axis. The assumption of constant rate of twist corresponds to assuming homogeneous St. Venant torsion with identical cross-sectional warping along the beam. As in the extension mode, no internal generalized shear forces $Q_{\alpha}$ occur which leads to zero in-plane displacement increment $\Delta u_{\alpha}\left(x_{\alpha}\right)$ for each node pair. The displacement increments defining the twist deformation mode then follow as

$$
\Delta u_{\alpha}\left(x_{\gamma}\right)=-e_{\alpha \beta} x_{\beta} \eta_{3}, \quad \Delta u_{3}\left(x_{\gamma}\right)=0, \quad \text { Node pairs } i=1, \ldots, m .
$$

\subsection{Bending modes}

The two bending deformation modes illustrated in Fig. 4(c) are characterized by a constant bending curvature about the in-plane coordinates $x_{\alpha}$ equal to $\eta_{\alpha}$. As no internal generalized 
shear forces $Q_{\alpha}$ occur in this mode, the in-plane displacement increment $\Delta u_{\alpha}\left(x_{\alpha}\right)$ for each node pair is zero. The displacement increments defining the two bending deformation modes then follow as

$$
\Delta u_{\alpha}\left(x_{\gamma}\right)=0, \quad \Delta u_{3}\left(x_{\gamma}\right)=\eta_{\alpha} e_{\alpha \beta} x_{\beta}, \quad \text { Node pairs } i=1, \ldots, m .
$$

\subsection{Shear modes}

The two shear deformation modes illustrated in Fig. 4(d) are characterised by the combination of a transverse shearing displacement and an antisymmetric deformation associated with the presence of linearly varying bending moments. The latter contribution can be calculated from the nodal displacements of the extension, twist, and bending deformation modes. The nodal displacements of these four deformation modes $\mathbf{v}_{j}$ where $j=3,4,5,6$ and their corresponding mid section internal force vectors without the null shear force components defined as $\mathbf{q}_{0 i}^{r}=\left[\begin{array}{llll}Q_{3}^{0} & M_{1}^{0} & M_{2}^{0} & M_{3}^{0}\end{array}\right]^{T}$ are arranged as columns in the following two matrices

$$
\mathbf{V}_{r}=\left[\mathbf{v}_{3}, \ldots, \mathbf{v}_{6}\right], \quad \mathbf{R}_{r}=\left[\mathbf{q}_{03}^{r}, \ldots, \mathbf{q}_{06}^{r}\right]
$$

A matrix containing the nodal displacements corresponding to unit equilibrium loads can then be obtained by post-multiplication by the matrix $\mathbf{R}_{r}^{-1}$,

$$
\mathbf{V}_{u}=\mathbf{V}_{r} \mathbf{R}_{r}^{-1}
$$

where the column vectors of the matrix $\mathbf{V}_{u}$ follow as

$$
\mathbf{V}_{u}=\left[\mathbf{v}_{3}^{u}, \ldots, \mathbf{v}_{6}^{u}\right]
$$

The column vectors $\mathbf{v}_{3}^{u}, \mathbf{v}_{4}^{u}, \mathbf{v}_{5}^{u}$, and $\mathbf{v}_{6}^{u}$ are the nodal displacements associated with the equilibrium states of tension, bending, and torsion for imposed unit load components.

The deformation associated with linearly varying bending moments along the beam can now be represented as a linear combination of the mid plane nodal displacements of the bending equilibrium states with unit equilibrium loads $\overline{\mathbf{v}}_{4}^{u}, \overline{\mathbf{v}}_{5}^{u}$,

$$
u_{j}^{a}\left(x_{k}\right)=\bar{u}_{j 4}^{u}\left(x_{\alpha}\right) M_{1}\left(x_{3}\right)+\bar{u}_{j 5}^{u}\left(x_{\alpha}\right) M_{2}\left(x_{3}\right), \quad \text { Node pairs } i=1, \ldots, m .
$$


Substituting the representation of the moments in terms of the shear forces according to (2) yields

$$
u_{j}^{a}\left(x_{k}\right)=x_{3}\left[-\bar{u}_{j 5}^{u}\left(x_{\alpha}\right) Q_{1}^{0}+\bar{u}_{j 4}^{u}\left(x_{\alpha}\right) Q_{2}^{0}\right], \quad \text { Node pairs } i=1, \ldots, m .
$$

Taking the difference in displacement across the beam, the nodal displacement increment from the presence of the linearly varying bending moments follows as

$$
\Delta u_{j}^{a}\left(x_{\alpha}\right)=l\left[-\bar{u}_{j 5}^{u}\left(x_{\alpha}\right) Q_{1}^{0}+\bar{u}_{j 4}^{u}\left(x_{\alpha}\right) Q_{2}^{0}\right], \quad \text { Node pairs } i=1, \ldots, m .
$$

The displacement increments which define the two shear deformation modes now follow as the summation of the contribution from the antisymmetric bending moment $\Delta u_{j}^{a}\left(x_{\alpha}\right)$ and the transverse shearing displacement defined by $\xi_{\alpha}$,

$$
\Delta u_{\alpha}\left(x_{\gamma}\right)=\xi_{\alpha}+\Delta u_{\alpha}^{a}\left(x_{\gamma}\right), \quad \Delta u_{3}\left(x_{\gamma}\right)=\Delta u_{3}^{a}\left(x_{\gamma}\right), \quad \text { Node pairs } i=1, \ldots, m
$$

\subsection{Rigid body motion}

Rigid body displacements must be constrained to completely define the kinematics associated with the six deformation modes. Since the beam is located in a $3 \mathrm{D}$ space, three rigid body rotations and three rigid body translations must be constrained. To ensure no generalized shear forces are present in the extension, twist, and bending deformation modes, constraints are imposed as to allow shearing of the beam. Shear deformation is tied with transverse displacement and inclination of the end faces. Constraining the increments in transverse displacements $\Delta u_{\alpha}\left(x_{\alpha}\right)=0$ for each node pair and not imposing constraints on the rotation about the $\alpha$-axis allows the beam to shear, while preventing rigid body rotation. The remaining rigid body rotation about the $x_{3}$-axis and the three rigid body translations can be constrained in terms of averages of the mean displacements

$$
\sum_{i=1}^{m} \bar{u}_{3 i}=0, \quad \sum_{i=1}^{m} \bar{u}_{\alpha i}=0, \quad \sum_{i=1}^{m} x_{\alpha i} e_{\alpha \beta} \bar{u}_{\beta i}=0,
$$

where $x_{\alpha i}$ and $\bar{u}_{j i}$ are the in-plane nodal coordinates and the three mean displacements of node pair $i$, respectively. 
For the two shear deformation modes, rigid body motion is constrained by ensuring that the deformation is orthogonal to the deformation in the other four modes. The orthogonality is expressed in terms of energy such that the displacements $\mathbf{v}_{1}, \mathbf{v}_{2}$ of the shear modes do not produce work with respect to the force distributions of the extension, twist and bending deformation modes,

$$
\mathbf{v}_{\alpha+}^{T} \mathbf{p}_{j+}-\mathbf{v}_{\alpha-}^{T} \mathbf{p}_{j-}=0, \quad \alpha=1,2 ; j=3,4,5,6 .
$$

The extension, twist and bending deformation modes have constant generalized internal forces, whereby the forces at the front and back face are of equal magnitude but with opposite sign, $\mathbf{p}_{+}=-\mathbf{p}_{-}$. Using this relation the orthogonality conditions simplify to

$$
\overline{\mathbf{v}}_{\alpha}^{T} \mathbf{p}_{j+}=0, \quad \alpha=1,2 ; j=3,4,5,6
$$

These relations clearly indicate that the orthogonality constraints act only on the mean displacements. From the nature of the deformation modes, the orthogonality conditions prevent all three rigid body rotations and rigid body translation in the $x_{3}$ direction. The remaining two rigid body translations in the $x_{\alpha}$ direction can be constrained in terms of averages of the mean displacements by use of the second formula in (32),

$$
\sum_{i=1}^{m} \bar{u}_{\alpha i}=0 .
$$

\section{Deformation modes using finite elements}

The properties of the cross-section are evaluated based on the global stiffness matrix of the slice obtained using the discretization method presented in Appendix A. The stiffness equations of the slice in terms of the nodal displacement at the front $(+)$ and back $(-)$ faces $\mathbf{v}_{ \pm}$take the following block matrix format

$$
\left[\begin{array}{ll}
\mathbf{K}_{++} & \mathbf{K}_{+-} \\
\mathbf{K}_{-+} & \mathbf{K}_{--}
\end{array}\right]\left[\begin{array}{l}
\mathbf{v}_{+} \\
\mathbf{v}_{-}
\end{array}\right]=\left[\begin{array}{l}
\mathbf{f}_{+} \\
\mathbf{f}_{-}
\end{array}\right]
$$


The stiffness equations in terms of the increment and mean of forces and displacements across the length of the beam follow as

$$
\left[\begin{array}{ll}
\mathbf{K}_{11} & \mathbf{K}_{12} \\
\mathbf{K}_{21} & \mathbf{K}_{22}
\end{array}\right]\left[\begin{array}{c}
\Delta \mathbf{v} \\
2 \overline{\mathbf{v}}
\end{array}\right]=\left[\begin{array}{c}
\mathbf{f}_{+}-\mathbf{f}_{-} \\
\mathbf{f}_{+}+\mathbf{f}_{-}
\end{array}\right]
$$

where the block matrices are defined as

$$
\begin{aligned}
& \mathbf{K}_{11}=\frac{1}{2}\left(\mathbf{K}_{++}-\mathbf{K}_{+-}-\mathbf{K}_{-+}+\mathbf{K}_{--}\right), \\
& \mathbf{K}_{12}=\frac{1}{2}\left(\mathbf{K}_{++}+\mathbf{K}_{+-}-\mathbf{K}_{-+}-\mathbf{K}_{--}\right), \\
& \mathbf{K}_{21}=\frac{1}{2}\left(\mathbf{K}_{++}-\mathbf{K}_{+-}+\mathbf{K}_{-+}-\mathbf{K}_{--}\right), \\
& \mathbf{K}_{22}=\frac{1}{2}\left(\mathbf{K}_{++}+\mathbf{K}_{+-}+\mathbf{K}_{-+}+\mathbf{K}_{--}\right) .
\end{aligned}
$$

The six deformation modes are imposed by representing the translation increments $\Delta \mathbf{u}_{j}$ for all node pairs in terms of the six component deformation mode vector $\boldsymbol{\zeta}$ as described in Section 3 . The corresponding axial derivatives $\Delta \mathbf{u}_{j}^{\prime}$ are kept as part of the finite element problem to be solved. This is accomplished by introducing the representation

$$
\Delta \mathbf{v}=\mathbf{A} \mathbf{w}+\mathbf{B} \mathbf{q}_{0}
$$

in which the six deformation components and the derivative increments are contained in the vector $\mathbf{w}=\left[\boldsymbol{\zeta}^{T}, \Delta u_{j, 1}^{\prime}, \cdots, \Delta u_{j, m}^{\prime}\right]^{T}$, while the second term adds the displacement increments associated with an antisymmetric bending moment defined in (30). Note, that the second term is proportional to the length $l$. The transformation matrix $\mathbf{A}$ and the antisymmetric bending moment contribution matrix $\mathbf{B}$ are defined by the block matrix format

$$
\mathbf{A}=\left[\begin{array}{cccc}
\mathbf{A}_{1} & \mathbf{J}_{1} & & \\
\vdots & & \ddots & \\
\mathbf{A}_{m} & & & \mathbf{J}_{m}
\end{array}\right], \quad \mathbf{B}=\left[\begin{array}{c}
\mathbf{B}_{1} \\
\vdots \\
\mathbf{B}_{m}
\end{array}\right]
$$


where the matrix $\mathbf{A}_{i}$ defining the displacement increments and the matrix $\mathbf{J}_{i}$ retaining the derivative increments are given as

$$
\mathbf{A}_{i}=\left[\begin{array}{cccccc}
0 & 0 & 0 & 0 & 0 & -x_{2} \\
0 & 1 & 0 & 0 & 0 & x_{1} \\
0 & 0 & 1 & x_{2} & -x_{1} & 0 \\
0 & 0 & 0 & 0 & 0 & 0 \\
0 & 0 & 0 & 0 & 0 & 0 \\
0 & 0 & 0 & 0 & 0 & 0
\end{array}\right]_{i} \quad \mathbf{J}_{i}=\left[\begin{array}{ccc}
0 & 0 & 0 \\
0 & 0 & 0 \\
0 & 0 & 0 \\
1 & 0 & 0 \\
0 & 1 & 0 \\
0 & 0 & 1
\end{array}\right]_{i}
$$

Based on (30) the antisymmetric moment contribution matrix $\mathbf{B}_{i}$ is defined in terms of the beam length $l$ and the mean nodal displacements from the two unit bending equilibrium states $\bar{u}_{j 4}^{u}$ and $\bar{u}_{j 5}^{u}$,

$$
\mathbf{B}_{i}=l\left[\begin{array}{cccccc}
-\bar{u}_{15}^{u} & \bar{u}_{14}^{u} & 0 & 0 & 0 & 0 \\
-\bar{u}_{25}^{u} & \bar{u}_{24}^{u} & 0 & 0 & 0 & 0 \\
-\bar{u}_{35}^{u} & \bar{u}_{34}^{u} & 0 & 0 & 0 & 0 \\
0 & 0 & 0 & 0 & 0 & 0 \\
0 & 0 & 0 & 0 & 0 & 0 \\
0 & 0 & 0 & 0 & 0 & 0
\end{array}\right]_{i}
$$

Elimination of $\Delta \mathbf{v}$ in (37) by (39) gives

$$
\left[\begin{array}{cc}
\mathbf{A}^{T} \mathbf{K}_{11} \mathbf{A} & \mathbf{A}^{T} \mathbf{K}_{12} \\
\mathbf{K}_{21} \mathbf{A} & \mathbf{K}_{22}
\end{array}\right]\left[\begin{array}{c}
\mathbf{w} \\
2 \overline{\mathbf{v}}
\end{array}\right]=\left[\begin{array}{c}
\mathbf{A}^{T}\left(\mathbf{f}_{+}-\mathbf{f}_{-}-\mathbf{K}_{11} \mathbf{B} \mathbf{q}_{0}\right) \\
\left.\left(\mathbf{f}_{+}+\mathbf{f}_{-}-\mathbf{K}_{21} \mathbf{B} \mathbf{q}_{0}\right)\right)
\end{array}\right]
$$

In this form, the forces associated with each of the six deformation components $\zeta$ contained in the vector $\mathbf{w}$ are equal to minus twice the mid-section generalized forces $\mathbf{q}_{0}$. 


\subsection{Enforcing deformation modes using constraints}

Activating each of the six deformation modes is accomplished by the addition of constraints which enforce relationships among the degrees of freedom. Two types of constraints are needed, namely constraints to fix the value of the six deformation components $\boldsymbol{\zeta}$, and constraints which prevent rigid body motion of the slice.

The constraints that define the value of the six deformation components $\boldsymbol{\zeta}$ provide the handle needed to set one degree of freedom to unity and all others to zero. This corresponds to activating one deformation mode, while setting the remaining five to zero. These constraints are defined as

$$
\zeta=\mathbf{g}_{j}
$$

where the vector $\mathbf{g}_{j}$ is used to select which degree of freedom to activate while setting the remaining five to zero, where the index $j=1, \ldots, 6$ specifies which deformation is activated, e.g. $\mathbf{g}_{3}=[0,0,1,0,0,0]^{T}$ for the extension case.

Two different sets of constraints to prevent rigid body motion are needed in the analysis, namely one for the extension, twist, and bending deformation modes and the other one for the two shear deformation modes. These constraints act on the mean displacements and can be expressed as

$$
\mathbf{G}_{n} \overline{\mathbf{u}}=\mathbf{0},
$$

where $\mathbf{G}_{n}$ is the rigid body constraint matrix where the index $n$ ranging from 1 to 2 specifies which of the two sets of constraints are enforced. From (32) the constraint matrix using average displacements when solving the extension, twist, and bending deformation modes takes the form

$$
\mathbf{G}_{1}=\left[\mathbf{G}_{1 i}, \ldots, \mathbf{G}_{1 m}\right]
$$


where the sub-matrices $\mathbf{G}_{1 i}$ containing the in-plane nodal coordinates for node $i$ are defined as

$$
\mathbf{G}_{1 i}=\left[\begin{array}{cccccc}
1 & 0 & 0 & 0 & 0 & 0 \\
0 & 1 & 0 & 0 & 0 & 0 \\
0 & 0 & 1 & 0 & 0 & 0 \\
-x_{2} & x_{1} & 0 & 0 & 0 & 0
\end{array}\right]_{i}, \quad i=1, \ldots, m
$$

The constraints of the shear deformation problems make use of the combined orthogonality and mean value conditions (34) and (35). These are combined in the constraint matrix

$$
\mathbf{G}_{2}=\left[\begin{array}{c}
\mathbf{G}_{2}^{u} \\
\mathbf{G}_{2}^{l}
\end{array}\right]
$$

in which the matrix $\mathbf{G}_{2}^{u}$ enforcing the orthogonality conditions is defined as

$$
\mathbf{G}_{2}^{u}=\left[\mathbf{p}_{3+}, \mathbf{p}_{4+}, \mathbf{p}_{5+}, \mathbf{p}_{6+}\right]^{T}
$$

The matrix $\mathbf{G}_{2}^{l}$ enforcing rigid body constraints using mean displacements is defined as

$$
\mathbf{G}_{2}^{l}=\left[\mathbf{G}_{2 i}, \ldots, \mathbf{G}_{2 n}\right]
$$

where the sub-matrices $\mathbf{G}_{2 i}$ take the form

$$
\mathbf{G}_{2 i}=\left[\begin{array}{cccccc}
1 & 0 & 0 & 0 & 0 & 0 \\
0 & 1 & 0 & 0 & 0 & 0
\end{array}\right]_{i}, \quad i=1, \ldots, m
$$

The constraints are added to the system of linear equations using the method of Lagrange multipliers where each constraint is enforced by solving for the associated Lagrange multiplier, acting as the force needed to impose the constraint, $[13,14]$. When no external forces are applied to the slice in addition to constraint forces, the Lagrange multipliers associated with the degrees of freedom of the deformation modes are the constraint forces, here in the form of minus twice the mid section generalized forces. This enables the antisymmetric moment contribution to be 
moved inside the stiffness matrix. Incorporating the constraints to define the six deformation components and the constraints to prevent rigid body motion and their associated Lagrange multipliers to be solved $\boldsymbol{\lambda}_{\zeta}$ and $\boldsymbol{\lambda}_{r}$, respectively, the system of equations of (43) takes the following form

$$
\left[\begin{array}{cccc}
\mathbf{A}^{T} \mathbf{K}_{11} \mathbf{A} & \mathbf{A}^{T} \mathbf{K}_{12} & \mathbf{L}^{T}-\frac{1}{2} \mathbf{A}^{T} \mathbf{K}_{11} \mathbf{B} & \mathbf{0} \\
\mathbf{K}_{21} \mathbf{A} & \mathbf{K}_{22} & -\frac{1}{2} \mathbf{K}_{21} \mathbf{B} & \mathbf{G}_{n}^{T} \\
\mathbf{L} & \mathbf{0} & \mathbf{0} & \mathbf{0} \\
\mathbf{0} & \mathbf{G}_{n} & \mathbf{0} & \mathbf{0}
\end{array}\right]\left[\begin{array}{c}
\mathbf{w} \\
2 \overline{\mathbf{u}} \\
\boldsymbol{\lambda}_{\zeta} \\
\boldsymbol{\lambda}_{r}
\end{array}\right]_{j}=\left[\begin{array}{c}
\mathbf{0} \\
\mathbf{0} \\
\mathbf{g}_{j} \\
\mathbf{0}
\end{array}\right]
$$

The matrix $\mathbf{L}$ used to select the deformation components $\zeta$ takes the form

$$
\mathbf{L}=\left[\begin{array}{ll}
\mathbf{I}_{6 \times 6} & \mathbf{0}_{6 \times 3 m}
\end{array}\right] .
$$

The form of the rigid body constraint matrix $\mathbf{G}_{n}$ depends on the analysis step, with $\mathbf{G}_{1}$ for the extension, torsion and bending modes and $\mathbf{G}_{2}$ for the two shear modes.

It is convenient to present (52) in a compact notation where the displacement and force terms are grouped together

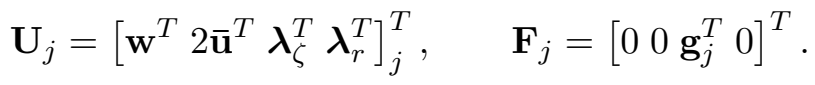

In this notation, the system of equations takes the form

$$
\mathbf{K}_{c} \mathbf{U}_{j}=\mathbf{F}_{j},
$$

in which the stiffness matrix supplemented by the constraint matrices is contained in the matrix $\mathbf{K}_{c}$.

\section{Cross-section stiffness matrix analysis procedure}

The cross-section stiffness analysis procedure is summarized in Table 1 in pseudo-code format. The first part of the analysis consists of assembling both the stiffness matrix of the slice in block format and the transformation matrix A based on (40). 
Table 1: Analysis procedure.

\begin{tabular}{|c|c|}
\hline & MAIN \\
\hline (1) & Initialize $\mathbf{g}_{j}, \mathbf{B}$ and $\mathbf{G}_{n}$ \\
\hline$(2)$ & Calculate matrices $\mathbf{K}_{11}, \mathbf{K}_{12}, \mathbf{K}_{21}, \mathbf{K}_{22}$, and $\mathbf{A}$ \\
\hline \multirow[t]{2}{*}{ (3) } & Deformation modes: \\
\hline & $\begin{array}{l}\text { Run MODES with } \mathbf{G}_{1} \text { for extension, bending and twist modes, } j=3,4,5,6 \\
\text { Update } \mathbf{B} \text { and } \mathbf{G}_{2} \\
\text { Run MODES with } \mathbf{G}_{2} \text { for shear modes } j=1,2\end{array}$ \\
\hline \multirow[t]{5}{*}{$(5)$} & Flexibility and stiffness matrices: \\
\hline & $\begin{array}{l}\mathbf{V}=\left[\mathbf{v}_{1}, \ldots, \mathbf{v}_{6}\right], \quad \mathbf{P}=\left[\mathbf{p}_{1}, \ldots, \mathbf{p}_{6}\right], \quad \mathbf{R}=\left[\mathbf{q}_{01}, \ldots, \mathbf{q}_{06}\right] \\
\mathbf{H}=\mathbf{R}^{-T}\left(\mathbf{V}^{T} \mathbf{P}\right) \mathbf{R}^{-1}\end{array}$ \\
\hline & $\begin{array}{l}\mathbf{H}=\mathbf{R}^{-1}\left(\mathbf{V}^{-1} \mathbf{P}\right) \mathbf{R}^{-1} \\
\mathbf{C} \text { from }(11)\end{array}$ \\
\hline & $\mathbf{D}=\mathbf{C}^{-1}$ \\
\hline & MODES \\
\hline (a) & $\mathbf{U}_{j}=\mathbf{K}_{c}^{-1} \mathbf{F}_{j}$ \\
\hline (b) & $\Delta \mathbf{v}=\mathbf{A} \mathbf{w}+\mathbf{B} \mathbf{q}_{0}$ \\
\hline (c) & $\mathbf{v}_{ \pm}=\frac{1}{2}(\Delta \mathbf{v} \pm \overline{\mathbf{v}})$ \\
\hline (d) & $\mathbf{p}_{j}=\mathbf{K} \mathbf{v}_{j}$ \\
\hline
\end{tabular}

The next step is to analyse the six deformation modes. First the extension, torsion and bending deformation modes are analysed using the rigid body constraint matrix $\mathbf{G}_{1}$ defined in (46). The nodal displacements $\mathbf{v}_{j}$ from these four deformation modes are used to populate the antisymmetric moment contribution matrix B based on (40), while the corresponding nodal force distributions $\mathbf{p}_{j}$ are used to create the constraint matrix $\mathbf{G}_{2}$ from (48). The two shear deformation modes are then calculated.

The nodal displacements, conjugate nodal forces and mid section force vectors of the six deformation modes are then arranged as columns in the matrices $\mathbf{V}, \mathbf{P}$ and $\mathbf{R}$, respectively. The beam flexibility matrix $\mathbf{H}$ can then be determined from (19). The cross-section flexibility matrix 
$\mathbf{C}$ is then calculated using (11) and finally the corresponding cross-section stiffness matrix $\mathbf{D}$ is obtained by inversion of this flexibility matrix.

\section{Examples}

This section contains an assessment of the capacity of the present method for calculating the full six by six set of stiffness coefficients of general cross-sections. Three examples are used to cover solid and thin-walled sections as well as isotropic and general anisotropic materials, namely an isotropic circular section, a rectangular section with antisymmetric composite layup, and a realistic wind turbine blade cross-section with off-axis fibers in the spar cap. It is noted, that for thin-walled, single-layer, orthotropic cross-sections the isoparametric representation used in [10] comes out as a special case of the present formulation, although set up in a different way as a two-dimensional theory for the cross-section.

\subsection{Isotropic circular section}

The first example concerns the circular cross-section geometry shown in Fig. 5(a), where the circle has a radius of $r=1$ and is made of an isotropic material with Poisson ratio $\nu=0.3$. The cross-section is discretized using $n$ layers in the radial direction and $4 n$ segments in the circumferential direction using elements with quadratic interpolation in the cross-section plane and Hermitian cubic interpolation in the axial direction. The case $n=2$ is shown in Fig. 5(b).

(a)

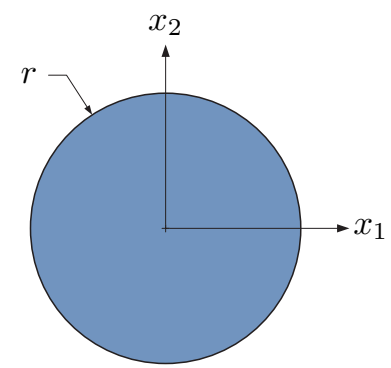

(b)

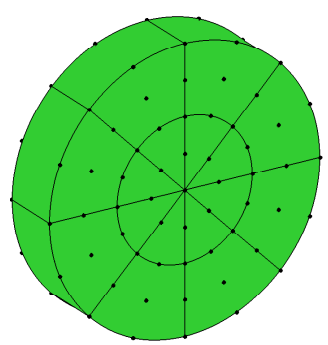

Figure 5: (a) Schematic of a circular section, (b) $n=2$ finite element discretization. 
Table 2: Normalized cross-section stiffness properties for an isotropic circle.

\begin{tabular}{lccccc}
\hline \multicolumn{5}{c}{ Mesh refinement parameter $\mathrm{n}$} \\
& 1 & 2 & 4 & 8 & Analytical \\
\hline$A / \pi r^{2}$ & 0.9882 & 0.9992 & 0.9999 & 1.0000 & 1.0000 \\
$I_{1} / \pi r^{4}=I_{2} / \pi r^{4}$ & 0.2468 & 0.2498 & 0.2500 & 0.2500 & 0.2500 \\
$J / \pi r^{4}$ & 0.4883 & 0.4992 & 0.5000 & 0.5000 & 0.5000 \\
$A_{1} / \pi r^{2}=A_{2} / \pi r^{2}$ & 0.8604 & 0.8504 & 0.8507 & 0.8507 & 0.8507 \\
\hline
\end{tabular}

The reference axis being at the center, only the diagonal terms in the stiffness matrix are nonzero. The diagonal terms are $G A_{1}, G A_{2}, E A, E I_{1}, E I_{2}$, and $G J$ representing the shear stiffness about both in-plane axes, the extension stiffness, the bending stiffness about both in-plane axes, and the torsion stiffness, respectively. From symmetry of the section $G A_{1}=G A_{2}$ and $E I_{1}=E I_{2}$. Results for isotropic cross-sections obtained using different mesh sizes and an analytical solution from Renton [15] are listed in Table 2. The relative error of the stiffness coefficients with respect to the analytical solution are plotted in Fig. 6, illustrating cubic convergence for all parameters towards the analytical solution. Two elements in the radial direction are required to obtain

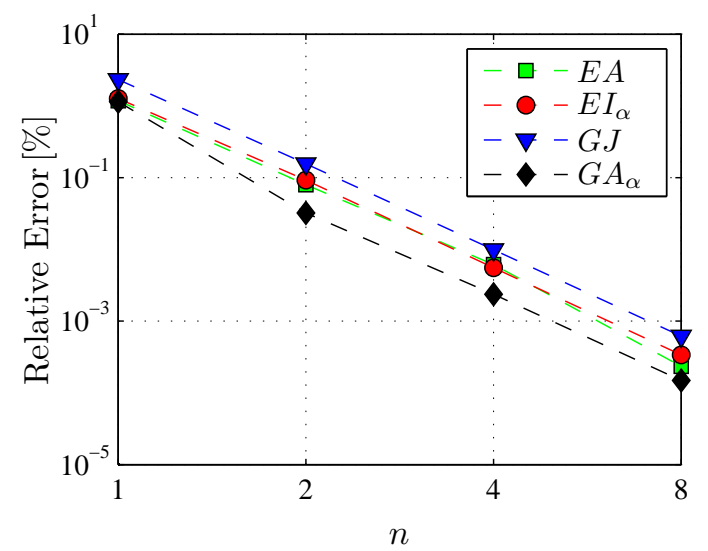

Figure 6: Relative error in stiffness coefficients with respect to the mesh refinement parameter. 
(a)

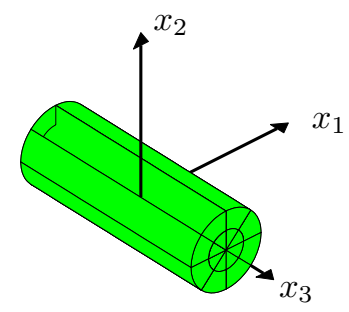

(d)

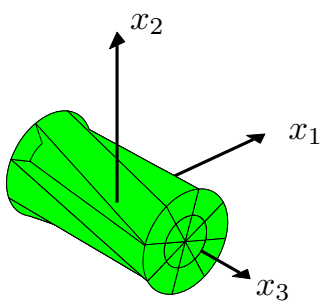

(b)

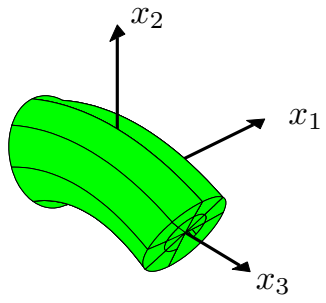

(e)

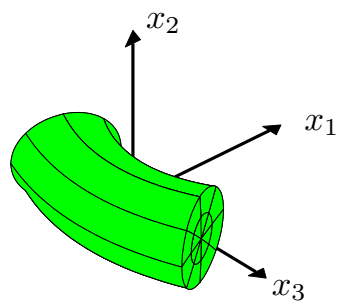

(c)

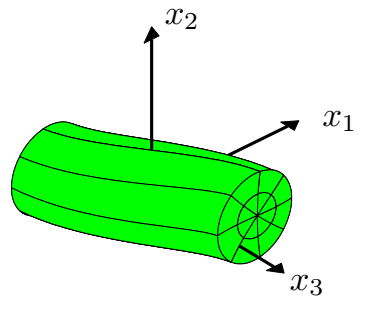

(f)

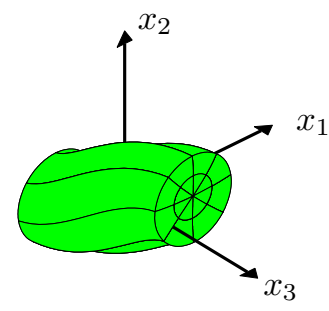

Figure 7: Deformation modes: (a) Extension, (d) Twist, (b,e) Bending, (c,f) Shear.

convergence of all the stiffness coefficients to within $1 \%$ relative error. This is due to the inability of four quadratic triangular elements to capture exactly the geometry of the circle as well as the occurrence of cubic terms in the cross-section coordinates in the transverse shear problem.

The 3D deformation of the six deformation modes are presented graphically in Fig. 7 using two layers of elements and a slice thickness of 2 to clearly illustrate the deformation modes. The quadratic curvature associated with the bending deformations is seen in Figs. 7(b) and (e). Furthermore in the two shear cases, the cubic displacement in the thickness direction is modelled with the use of a single element in the thickness direction via the Hermitian interpolation. It can be seen in Figs. 7(d) that the twist mode does not develop an axial warping due to the circular cross-section. The extension, bending and twist deformation modes exhibit constant in-plane contractions along the axial direction, whereas the shear modes have varying in-plane displacements because of the presence of linearly varying bending moments. 


\subsection{Composite rectangular section}

The second example considers the solid rectangular section made of layered orthotropic material as shown in Fig. 8(a) with width of $2 a=0.05 \mathrm{~m}$ and height of $2 b=0.1 \mathrm{~m}$. The upper and lower halves of the section are made of composite material with properties taken from [3], namely $E_{i}=141.963 \mathrm{GPa}, E_{j}=E_{k}=9.79056 \mathrm{GPa}, G_{i j}=G_{i k}=G_{j k}=59.9844 \mathrm{GPa}$ and $\nu_{i j}=\nu_{i k}=\nu_{j k}=0.42$, where the $i j k$ system constitutes the principal material directions in the plane of the lamina, as shown in Fig. 8(b). Moreover, the $x_{i} x_{j}$-plane is in the plane of the lamina and parallel to the $x_{3} x_{1}$-plane while the $x_{i}$ axis is along the fiber direction. The fiber orientation angle $\theta$ is defined as the angle between the $x_{3}$ axis and the $x_{i}$ axis.

(a)

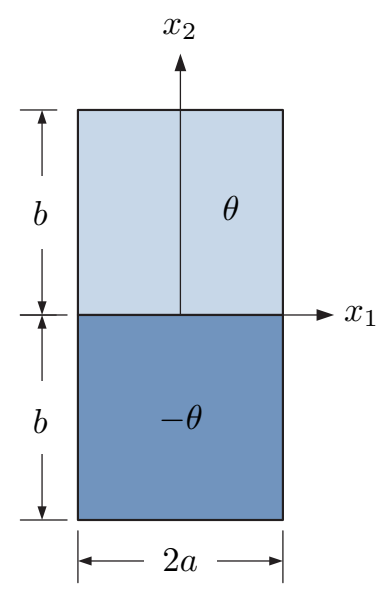

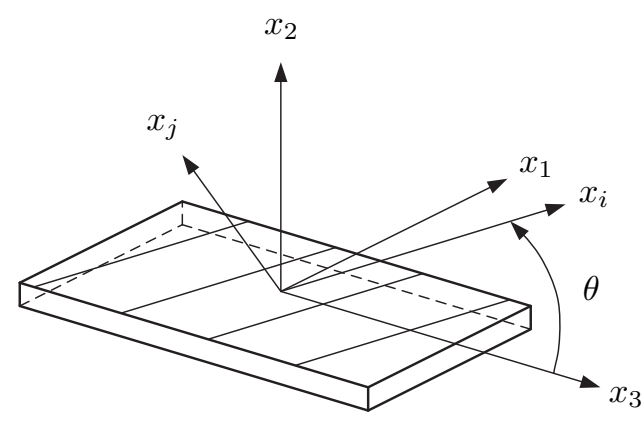

(b)

Figure 8: (a) Schematic of a composite rectangular section, (b) Definition of principal material axes.

The cross-section parameters of the composite rectangular section with $\theta=45^{\circ}$ using a regular mesh of $10 \times 10$ elements with quadratic interpolation in the cross-section plane and Hermitian cubic interpolation in the axial direction are presented in Table 3. The results are compared to the values obtained using the commercial code VABS using 744 quadratic elements. It is observed that for this section, results match well those calculated using VABS, with the maximum percentage difference $(0.25 \%)$ occurring for the shear stiffness in the $x_{1}$ direction. This 


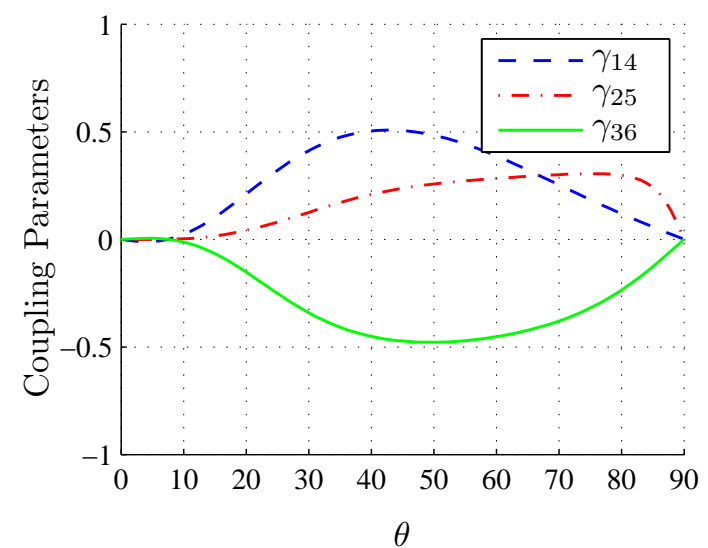

Figure 9: Coupling parameters as function of the fiber orientation angle $\theta$.

configuration exhibits strong shear-bending coupling and extension-torsion coupling, and the torsional rigidity is an order of magnitude less than the coupling. A measure of the importance of the off-diagonal terms can be obtained by normalizing them with respect to their associated diagonal terms,

$$
\gamma_{i j}=\frac{D_{i j}}{\sqrt{D_{i i} D_{j j}}}
$$

Table 3: Cross-section stiffness properties for the composite rectangular section with $\theta=45^{\circ}$.

\begin{tabular}{ccccc}
\hline & Units & VABS & Mesh 10x10 & \% Diff. \\
\hline$G A_{1}$ & {$[\mathrm{~N}]$} & $5.395 \mathrm{E}+07$ & $5.409 \mathrm{E}+07$ & 0.25 \\
$G A_{2}$ & {$[\mathrm{~N}]$} & $9.660 \mathrm{E}+07$ & $9.659 \mathrm{E}+07$ & 0.01 \\
$E A$ & {$[\mathrm{~N}]$} & $2.955 \mathrm{E}+08$ & $2.957 \mathrm{E}+08$ & 0.05 \\
$E I_{1}$ & {$\left[\mathrm{Nm}^{2}\right]$} & $2.434 \mathrm{E}+05$ & $2.436 \mathrm{E}+05$ & 0.06 \\
$E I_{2}$ & {$\left[\mathrm{Nm}^{2}\right]$} & $4.945 \mathrm{E}+04$ & $4.955 \mathrm{E}+04$ & 0.19 \\
$G J$ & {$\left[\mathrm{Nm}^{2}\right]$} & $1.326 \mathrm{E}+05$ & $1.326 \mathrm{E}+05$ & 0.01 \\
$D_{14}$ & {$[\mathrm{Nm}]$} & $1.832 \mathrm{E}+06$ & $1.836 \mathrm{E}+06$ & 0.21 \\
$D_{25}$ & {$[\mathrm{Nm}]$} & $5.198 \mathrm{E}+05$ & $5.197 \mathrm{E}+05$ & 0.03 \\
$D_{36}$ & {$[\mathrm{Nm}]$} & $-2.960 \mathrm{E}+06$ & $-2.960 \mathrm{E}+06$ & 0.00 \\
\hline
\end{tabular}


(a)

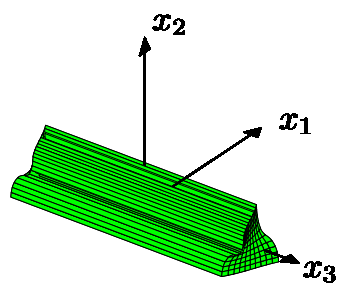

(d)

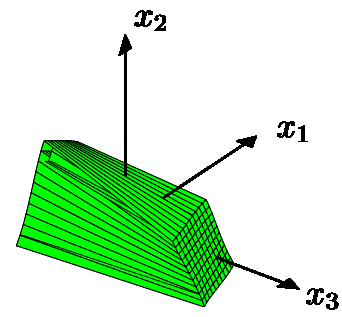

(b)

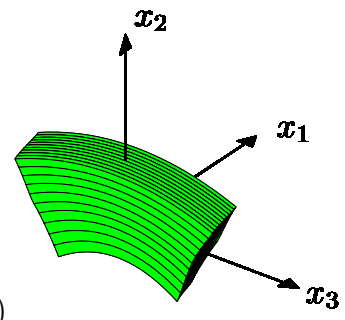

(e)

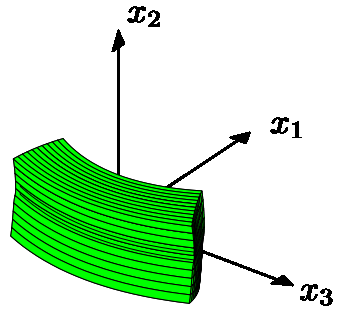

(f)

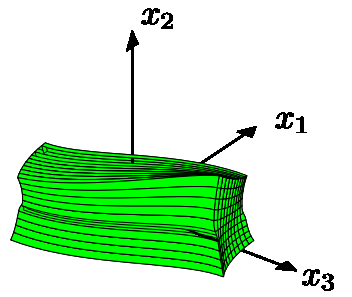

(c)

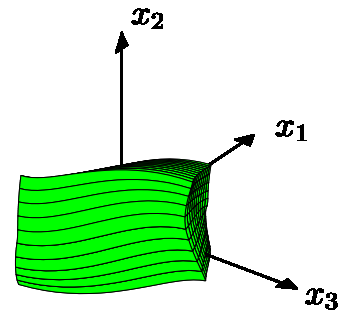

Figure 10: Deformation modes of the composite rectangular section: (a) Extension, (d) Twist, (b,e) Bending, $(\mathrm{c}, \mathrm{f})$ Shear.

The non-dimensional coupling parameters $\gamma_{i j}$ range from $-1<\gamma_{i j}<1$ where the extreme values indicate maximum possible coupling. Figure 9 shows the value of the coupling parameters with respect to the ply angle. It is seen that the maximum value for each coupling does not occur for the same fiber orientation angle, with the maximum for the shear-bending coupling $\gamma_{14}$ occurring at $43^{\circ}$, for the shear-bending coupling $\gamma_{25}$ at $75^{\circ}$, and for the extension-torsion coupling $\gamma_{36}$ at $50^{\circ}$.

The deformation modes are shown graphically in Fig. 10. It is seen that the extension, bending, and torsion modes have a uniform transverse deformation along the $x_{3}$ axis. Furthermore, shearing occurs in these four modes because of the presence of off-axis fibers.

\subsection{Wind turbine blade section}

A concept developed in the late 1980's for reducing loads seen by wind turbines is to promote blade twisting to decrease the angle of attack when subject to a wind gust with the use of biased 
layups in the blade spar cap [16, 17]. This final example of the present paper concerns the analysis of the two-cell cross-section of a wind turbine blade shown in Fig. 11 that exhibits bend-twist coupling via the use off-axis fibers in the spar cap. The section has a $1 \mathrm{~m}$ chord and the outer geometry of a S825 airfoil which is normally used at $75 \%$ of the blade radius. The origin of the coordinate system is placed at the leading edge of the section and the $x_{1}$ axis is co-linear with the airfoil chord line. The spar cap extends from $0.13 \mathrm{~m}$ to $0.47 \mathrm{~m}$ with the shear web positioned vertically at the center of the spar cap at $0.30 \mathrm{~m}$. The material properties are taken from Griffin [18]. The two different material layups used in the cross section are listed in Table 4. Note, that the thickness of the triaxial fabric has been modified for ease of modelling. Each section is made of three laminas having the triaxial fabric on the outer and inner surfaces and a balsa core for the skin and web sections and a spar cap mixture core for the spar cap sections. The spar cap mixture is made of alternate layers of triaxial fabric and uniaxial fabric. The stiffness properties for each layup are listed in Table 5 . Because the shear stiffness $G_{23}$ was not provided in the reference, it was calculated assuming transversely isotropic composites.

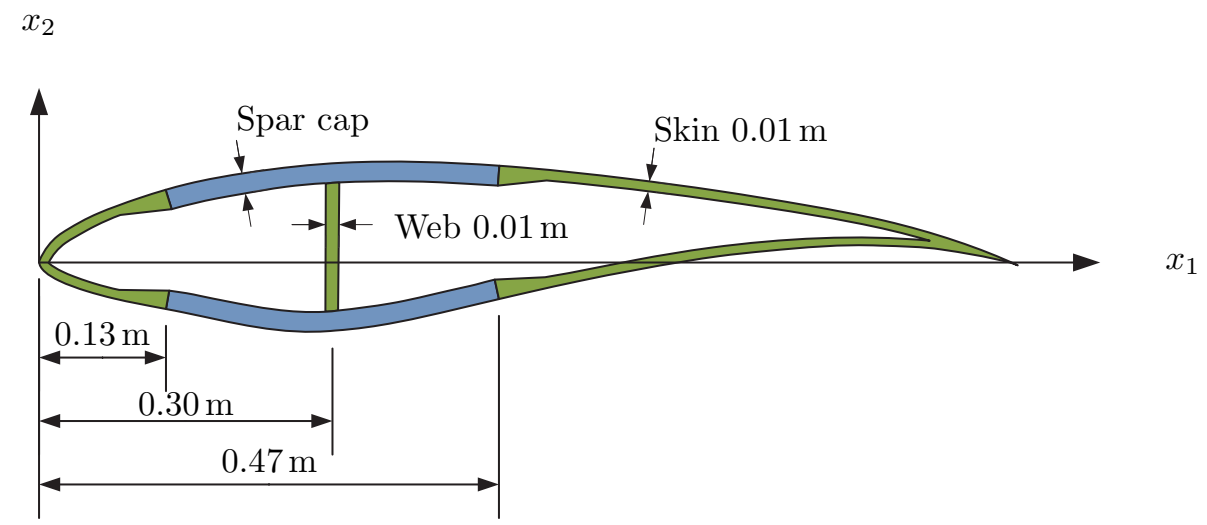

Figure 11: Schematic of a wind turbine blade section.

Previous work by Høgsberg and Krenk [10] has shown that extensive flanges and parts of thin-walled structures can be effectively modelled using elements with cubic-linear interpolations 
Table 4: Cross-section layup definition.

\begin{tabular}{lcc}
\hline Section & Material & Thickness $[\mathrm{mm}]$ \\
\hline Skin \& Web & triaxial fabric & 1.5 \\
& balsa & 7.0 \\
& triaxial fabric & 1.5 \\
\hline \multirow{2}{*}{ Spar Cap } & triaxial fabric & 3.5 \\
& spar cap mixture & user defined \\
& triaxial fabric & 3.5 \\
\hline
\end{tabular}

in the cross-section plane. Figure 12 shows the mesh of the wind turbine cross-section used in the current analysis obtained using cubic-linear elements. The skin, web and spar cap are modelled using thirteen 16-node elements with cubic-linear interpolation in the cross-section plane and Hermitian cubic interpolation in the axial direction. The trailing edge and transitions between sections with different thicknesses are modelled using eight 8-node elements with linear interpolation in the cross-section plane and Hermitian cubic interpolation in the axial direction. The discretization of the slice contains a total of 184 nodes.

The effect of the thickness and material orientation of the spar cap on the bending stiffness about the $x_{1}$ axis $E I_{1}$ and the bend-twist coupling parameter $\gamma_{46}$ is shown in Fig. 13. The spar cap thickness was varied from $20 \mathrm{~mm}$ to $35 \mathrm{~mm}$ and the material orientation from $0^{\circ}$ to $-45^{\circ}$, where the material orientation follows from Fig. 8(b). A maximum bend-twist coupling

Table 5: Material properties.

\begin{tabular}{lccccc}
\hline Material & $E_{11}[\mathrm{GPa}]$ & $E_{22}=E_{33}[\mathrm{GPa}]$ & $G_{12}=G_{13}[\mathrm{GPa}]$ & $G_{23}[\mathrm{GPa}]$ & $\nu_{12}=\nu_{13}=\nu_{23}$ \\
\hline Balsa & 2.07 & 2.07 & 0.14 & 0.863 & 0.22 \\
Spar cap mixture & 27.1 & 8.35 & 4.70 & 3.05 & 0.37 \\
Triaxial fabric & 24.2 & 8.97 & 4.97 & 3.23 & 0.39 \\
\hline
\end{tabular}




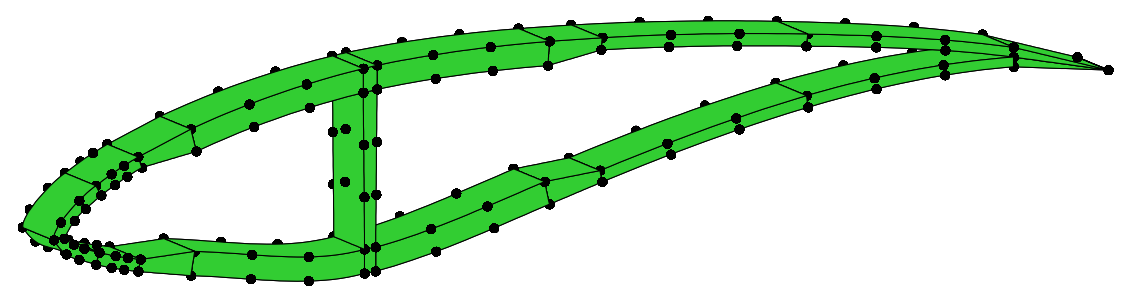

Figure 12: Finite element discretization of blade section.

parameter value of $\gamma_{46}=0.068$ is obtained with a material orientation of $\theta=-30^{\circ}$. It can be seen in Fig. 13(b) that the coupling parameter is rather insensitive to the spar cap thickness. Conversely, from Fig. 13(a) it is seen that both the spar cap thickness and fiber orientation have an effect on the bending stiffness, where a smaller material angle and thicker spar cap yield larger bending stiffness. The bending stiffness varies almost linearly with the thickness for the range studied. The bending stiffness is less sensitive to changes in material orientation close to $\theta=0^{\circ}$. This is expected from stress and strain coordinate transformation, where the axial stiffness component varies with the cosine of the angle while the shear-extension stiffness
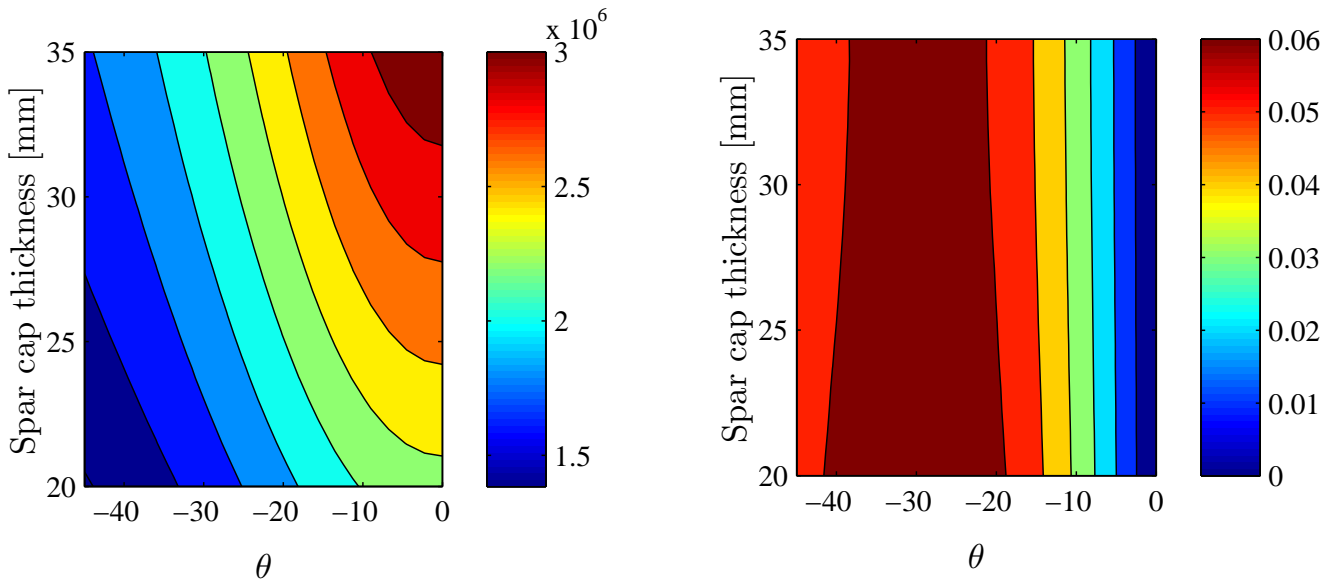

Figure 13: Effect of varying spar cap thickness and spar cap fiber orientation:(a) Bending stiffness $E I_{1}$, (b) Bend-twist coupling $\gamma_{46}$. 
component varies with the sine of the angle. Small material angles can therefore be used to obtain bend-twist coupling while limiting the increase in spar cap thickness needed to maintain a constant bending stiffness. For example, a $-7^{\circ}$ material orientation would yield a coupling parameter of $\gamma_{46}=0.027$ and would only require a $4 \%$ increase in the spar cap thickness to maintain the same bending stiffness as a configuration with no off-axis fibers (i.e. $0^{\circ}$ ) and a $20 \mathrm{~mm}$ spar cap. Doubling the value of the coupling parameter would require a $-16^{\circ}$ material orientation and an increase in thickness of $22 \%$ to maintain the same bending stiffness.

\section{Conclusions}

A method for analysing the cross-section stiffness properties of elastic beams with arbitrary cross-section geometry and material distribution has been presented. The method is based on analysis of a slice of the beam on which six independent deformation modes corresponding to extension, torsion, bending and shear are prescribed by imposing displacement increments across the slice via Lagrange multipliers. The six by six cross-section flexibility matrix is obtained using complementary elastic energy calculated in terms of the internal force/moment components and then inverted to give the cross-section stiffness matrix. The slice is modelled using a single layer of 3D finite elements with standard discretization in the cross-section plane and Hermitian cubic interpolation in the length-wise direction, whereby the degrees of freedom are concentrated on the front and back faces of the slice.

Three examples of increasing complexity have been used to demonstrate the accuracy of the procedure for a number of cases including both solid and thin-walled sections and isotropic and general anisotropic materials. Results show excellent agreement with analytical solutions and values predicted by the commercial code VABS. Finally, the method has been used to illustrate the compromise between bend-twist coupling and bending stiffness of a realistic cross-section of a wind turbine blade with off-axis fibers in its spar-cap. 


\section{Acknowledgment}

This work has been supported by Siemens Wind Power A/S.

\section{References}

[1] D. H. Hodges, Review of composite rotor blades modeling, AIAA Journal, 2 (1990) 561-565.

[2] S. N. Jung, V. T. Nagaraj, I. Chopra, Assessment of composite rotor blade modeling techniques, Journal of the American Helicopter Society, 44 (1999) 188-205.

[3] H. Chen, W. Yu, and M. Capellaro, A critical assessment of computer tools for calculating composite wind turbine blade properties, Wind Energy, 13 (2010) 497-516.

[4] D. H. Hodges, Nonlinear Composite Beam Theory, Progress in Astronautics and Aeronautics, AIAA, Virginia, 2006.

[5] D. Hodges, W. Yu. A rigorous, engineer friendly approach for modelling realistic, composite rotor blades, Wind Energy 10 (2007) 179-193.

[6] V. Giavotto, M. Borri, P. Mantegazza, G. Ghiringhelli, V. Carmaschi, G. C. Maffioli and F. Mussi. Anisotropic beam theory and applications, Computers and Structures 16 (1983) 497-516.

[7] G. L. Ghiringhelli, P. Mantegazza, Linear, straight and untwisted anisotropic beam section properties from solid finite elements, Composites Engineering 4 (1994) 1225-1239.

[8] J.P. Blasques, User's Manual for BECAS. A cross-section analysis tool for anisotropic and inhomogeneous beam sections of arbitrary geometry, Technical report: RIS $\varnothing-R 1785$, DTU Wind Energy, Technical University of Denmark, Roskilde, Denmark, 2012.

[9] S. Krenk, B. Jeppesen, Finite elements for beam cross-sections of moderate wall thickness, Computers and Structures, 321989 1035-1043. 
[10] J. Høgsberg, S. Krenk, Analysis of moderately thin-walled beam cross-sections by cubic isoparametric elements, Computers and Structures, 134 (2014) 88-101.

[11] Y. Jonnalagadda, J.D. Whitcomb, Calculation of effective section properties for wind turbine blades, Wind Energy, 17 (2014) 297-316.

[12] S. Krenk, Element stiffness matrix for beams with general cross-section properties, Technical University of Denmark, Department of Mechanical Engineering, (2006).

[13] R. D. Cook, D. S. Malkus, M. E. Pleasha, R. J. Witt, Concepts and Applications of Finite Element Analysis, 4th ed., Wiley, New York, 2002.

[14] K. J. Bathe, Finite Element Procedures, Prentice-Hall, Englewood Cliffs, N.J. 1996.

[15] J. D. Renton, Generalized beam theory applied to shear stiffness, International Journal of Solids and Structures 27 (1991) 1955-1967.

[16] D. W. Lobitz, P. S. Veers, G. R. Eisler, D. J. Laino, P. G. Migliore, G. Bir, The use of twist-coupled blades to enhance the performance of horizontal axis wind turbines, Sandia National Laboratories, SAND 2001-1303, (2001).

[17] D. W. Lobitz and P. S. Veers, Load mitigation with bending/twist-coupled blades on rotors using modern control strategies, Wind Energy 6 (2003) 105-117.

[18] D. A. Griffin, WindPACT turbine design scaling studies technical area 1 - composite blades for 80- to 120-meter rotor. National Renewable Energy Laboratory, NREL/SR-500-29492, (2001).

\section{Appendix A. Finite element representation of a slice}

In the present analysis the beam is discretized using three-dimensional isoparametric finite elements with a Hermitian interpolation in the longitudinal direction $x_{3}$. The Hermitian in- 
terpolation provides the required third degree polynomial representation of the displacement field as well as places all the nodes on the front and back face of the beam slice, simplifying the definition of the deformation modes. To avoid ill-conditioned elements, a slice of thickness comparable to the in-plane element dimensions is used.

The theory of the finite element method is well established, see e.g. [13, 14], and the focus here is on the modifications in the interpolation method needed for Hermitian interpolation in the axial direction. The elements are described in terms of the intrinsic coordinates $\boldsymbol{\xi}=[\xi, \eta, \zeta]^{T}$ where the coordinate $\zeta$ is collinear with the global axial coordinate $x_{3}$. For hexahedron elements, the intrinsic coordinates cover the range $-1 \leq \xi, \eta, \zeta \leq 1$. An 8 -node element in the intrinsic coordinate system is shown in Fig. A.1. For this element, nodes 1-8 represent the corner nodes.

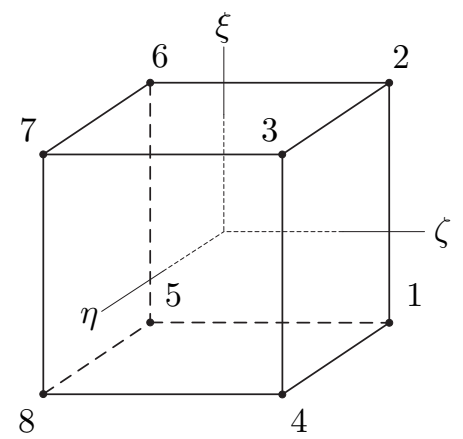

Figure A.1: 8-node element in intrinsic $\{\xi, \eta, \zeta\}$ coordinate system.

The displacement vector field $\mathbf{u}(\mathbf{x})=\left[u_{1}, u_{2}, u_{3}\right]^{T}$ is described using the representation

$$
\mathbf{u}(\mathbf{x})=\mathbf{N}(\boldsymbol{\xi}) \mathbf{v}=\sum_{j=1}^{2 m} \mathbf{N}_{j}(\boldsymbol{\xi}) \tilde{\mathbf{v}}_{j}
$$

where the shape functions associated with the nodal degrees of freedom $\tilde{\mathbf{v}}_{j}$ are contained in the 
Table A.1: Shape functions of 8-node element with Hermitian interpolation.

$$
\begin{aligned}
& \text { Corner nodes: } \xi_{i}= \pm 1, \eta_{i}= \pm 1, \zeta_{i}= \pm 1 \\
& N=\frac{1}{16}\left(1+\zeta_{i} \zeta\right)^{2}\left(2-\zeta_{i} \zeta\right)\left(1+\eta_{i} \eta\right)\left(1+\xi_{i} \xi\right) \\
& \text { Corner nodes: } \xi_{i}= \pm 1, \eta_{i}= \pm 1, \zeta_{i}= \pm 1 \\
& N_{h}=\frac{l}{32}\left(\zeta+\zeta_{i}\right)^{2}\left(\zeta-\zeta_{i}\right)\left(1+\eta_{i} \eta\right)\left(1+\xi_{i} \xi\right)
\end{aligned}
$$

block matrix $\mathbf{N}_{j}(\boldsymbol{\xi})$,

$$
\mathbf{N}_{j}(\boldsymbol{\xi})=\left[\begin{array}{cccccc}
N(\boldsymbol{\xi}) & 0 & 0 & N_{h}(\boldsymbol{\xi}) & 0 & 0 \\
0 & N(\boldsymbol{\xi}) & 0 & 0 & N_{h}(\boldsymbol{\xi}) & 0 \\
0 & 0 & N(\boldsymbol{\xi}) & 0 & 0 & N_{h}(\boldsymbol{\xi})
\end{array}\right]_{j}
$$

in which the shape functions associated with the vector components of the displacement field are labelled as $N$ and the shape functions associated with the displacement gradients are labelled as $N_{h}$.
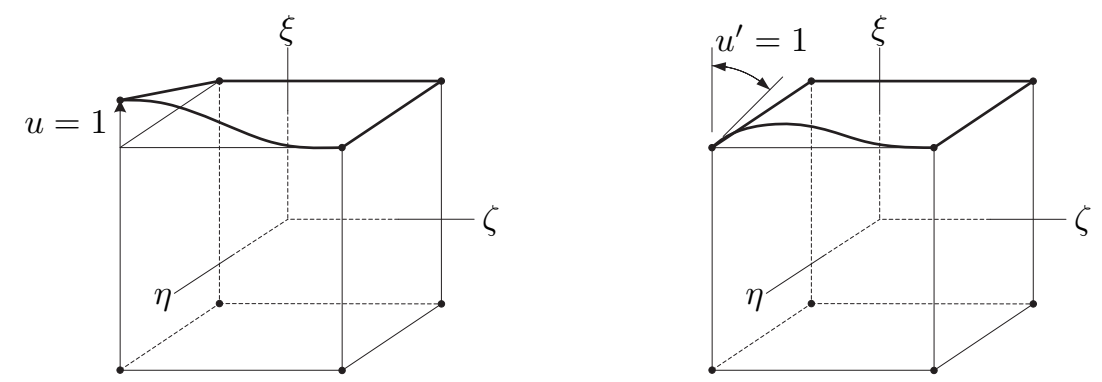

Figure A.2: Shape functions for 8-node element associated with corner node $\xi_{i}=1, \eta_{i}=1, \zeta_{i}=-1$.

The shape functions for an 8-node element with Hermitian interpolation are given in Table A.1. They are constructed using Lagrange product formulas. Shape functions associated with the corner node 7 are shown in Fig. A.2. The element is characterized by a linear variation in 


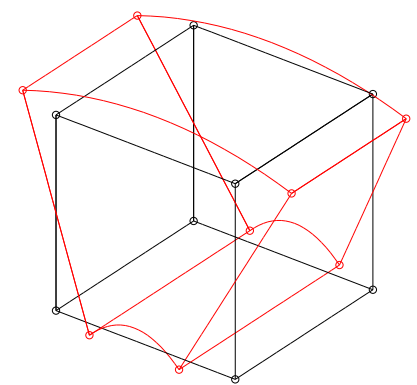

Figure A.3: Bending deformation of 8-node element with Hermitian interpolation, undeformed(-) deformed(-).

the $\xi, \eta$ plane and a cubic variation in $\zeta$. It should be noted that shape functions $N_{h}$ associated with the gradients of the displacement field are made proportional to the thickness of the slice, $l$, in order to have the gradients defined in the global coordinate system. The bending deformation of such an element is shown in Fig. A.3. The Hermitian interpolation enables capturing the quadratic displacement without intermediate nodes, and similarly for the cubic variation in the shear problem. 